\title{
Impurity resistivity of the double-donor system Si:P,Bi
}

\author{
A. Ferreira da Silva \\ Instituto Nacional de Pesquisas Espaciais, INPE, Laboratório Associado de Sensores e Materiais, LAS, C. P. 515, \\ 12201-970 São José dos Campos, SP, Brazil \\ Bo E. Sernelius \\ Department of Physics and Measurement Technology, Linköping University, S-58183 Linköping, Sweden \\ J. P. de Souza and H. Boudinov \\ Instituto de Física, Universidade Federal do Rio Grande do Sul, 91501-970 Porto Alegre, RS, Brazil \\ Hairong Zheng and M. P. Sarachik \\ Physics Department, City College of the City University of New York, New York, New York 10031
}

(Received 2 July 1999)

\begin{abstract}
The electrical resistivity of the shallow double-donor system Si:P,Bi, prepared by ion implantation, was investigated in the temperature range from 1.7 to $300 \mathrm{~K}$. Good agreement was obtained between the measured resistivities and resistivities calculated by a generalized Drude approach for the same temperatures and dopant concentrations. The critical impurity concentration for the metal-nonmetal transition for the double-doped $\mathrm{Si}: \mathrm{P}, \mathrm{Bi}$ system was found to lie between the critical concentrations of the two single-doped systems, Si:P and Si:Bi. [S0163-1829(99)11747-8]
\end{abstract}

\section{INTRODUCTION}

Double-doped semiconductors continue to be a subject of interest in the physics of disordered materials. In addition to the disorder due to the random spatial distribution of the dopant atoms, there is additional disorder in these systems associated with the different impurity ionization energies of the two dopant elements. In their pioneering investigation of a double-donor system, Newman and Holcomb ${ }^{1}$ used conductivity measurements to determine the critical concentration for the metal-nonmetal (MNM) transition in $\mathrm{Si}: \mathrm{P}, \mathrm{As}$. They showed that, rather than shifting to a higher value due to the additional energy disorder introduced by the double doping, the critical concentration occurs instead at the same reduced concentration as the two single donor materials, $\mathrm{Si}: \mathrm{P}$ and Si:As.

Silicon doped with bismuth is of particular interest because of its much larger ionization energy (smaller effective Bohr radius) compared to that of other group- $\mathrm{V}$ elements $(\mathrm{P}$, $\mathrm{As}$, and $\mathrm{Sb})^{2,3}$. Bearing in mind the recent investigation on transport properties of $\mathrm{Si}: \mathrm{Bi}^{3}{ }^{3}$ we investigate in this work the resistivity of the double donor system $\mathrm{Si}: \mathrm{P}, \mathrm{Bi}$ from room temperature down to $1.7 \mathrm{~K}$ for impurity concentrations ranging from the insulating to the metallic range.

The samples were prepared by ion implantation in van der Pauw $^{4}$ structures delineated in Si chips. Ion implantation is an essential process for the fabrication of electronic devices and integrated circuits. ${ }^{5-8}$ The resistivities obtained experimentally are compared with resistivity values calculated from a recently proposed generalized Drude approach ${ }^{3,9}$ (GDA) for single-doped systems, and here extended to double-doped systems at similar temperatures and dopant concentrations. The impurity critical concentration $N_{c}$ for the MNM transition is estimated from these results and calcu- lated using the dielectric function model with a LorentzLorenz correction.

\section{EXPERIMENTAL DETAILS}

Silicon wafers of $p$-type and (100) orientation with roomtemperature resistivities in the range of $11-25 \Omega-\mathrm{cm}$ were used for the fabrication of van der Pauw devices. The ohmic contact to the devices were phosphorus doped with low sheet resistance $(15 \Omega / \square)$.

Multiple implantation steps of $\mathrm{Bi}$ and $\mathrm{P}$ ions were accumulated in the devices in order to create similar plateaulike concentration depth profiles for $\mathrm{Bi}$ and $\mathrm{P}$ atoms, extending from the surface to a depth of $0.25 \mu \mathrm{m}$, with an estimated deviation of $\approx 5$ percent. The doses and energies of the implantation steps were determined from TRIM simulation. All the implants were carried out at nominal room temperature with the sample surface normal tilted at $7^{\circ}$ with respect to the direction of incidence of the beam in order to minimize channeling effects. The implantation steps were performed sequentially from the highest to the lowest energy, with the $\mathrm{Bi}^{-}$implants made prior to those of $\mathrm{P}^{+}$. Van der Pauw devices with donor atom concentrations $\left(N_{d}\right)$ at the plateaus of $1 \times 10^{20}, 5 \times 10^{19}, 1.0 \times 10^{19}, 8 \times 10^{18}, 7 \times 10^{18}, 6 \times 10^{18}, 4$ $\times 10^{18}$, and $5 \times 10^{17} \mathrm{~cm}^{-3}$ were prepared. For $N_{d}$ of 1 $\times 10^{20} \mathrm{~cm}^{-2}$, i.e., superposed plateaus of $5 \times 10^{19}$ of $\mathrm{Bi}$ and $5 \times 10^{19} \mathrm{~cm}^{-2}$ of $\mathrm{P}$, the following implantation steps were performed to the doses and energies, respectively of:

(a) $\mathrm{Bi}^{+}$at $4.9 \times 10^{14} \mathrm{~cm}^{-2} / 900 \mathrm{keV} ; 2.9 \times 10^{14} \mathrm{~cm}^{-2} / 550$ $\mathrm{keV} ; 2.0 \times 10^{14} \mathrm{~cm}^{-2} / 360 \mathrm{keV} ; 1.5 \times 10^{14} \mathrm{~cm}^{-2} / 200 \mathrm{keV}$; $8.0 \times 10^{13} \mathrm{~cm}^{-2} / 120 \mathrm{keV} ; 7.0 \times 10^{13} \mathrm{~cm}^{-2} / 70 \mathrm{keV} ; 5.0$ $\times 10^{13} \mathrm{~cm}^{-2} / 35 \mathrm{keV}$; and:

(b) $\mathrm{P}^{+}$at $7.0 \times 10^{14} \mathrm{~cm}^{-2} / 180 \mathrm{keV} ; 2.7 \times 10^{14} \mathrm{~cm}^{-2} / 120$ $\mathrm{keV} ; 1.8 \times 10^{14} \mathrm{~cm}^{-2} / 80 \mathrm{keV} ; 1.5 \times 10^{14} \mathrm{~cm}^{-2} / 55 \mathrm{keV}$; 


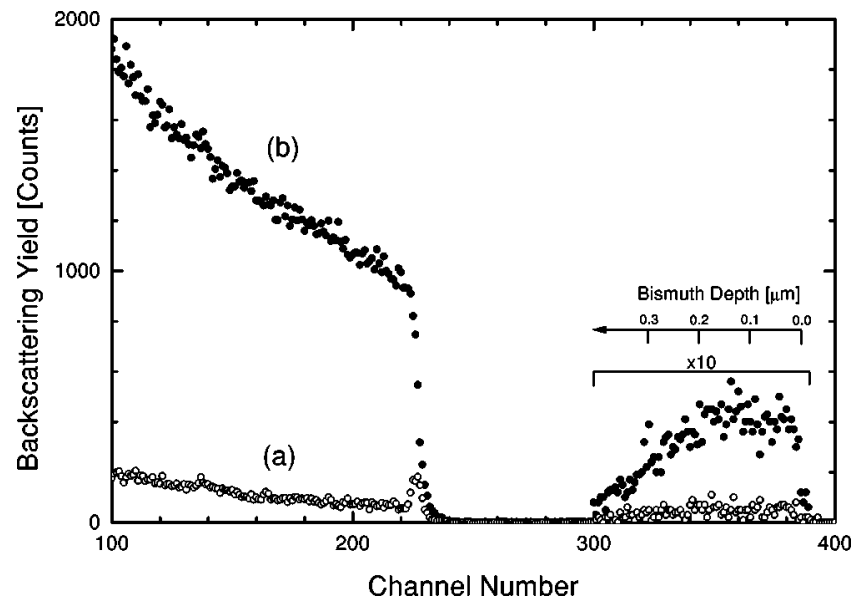

FIG. 1. Rutherford backscattering (RBS) spectra of the sample implanted to the highest dose $\left(N_{d}=1.0 \times 10^{20} \mathrm{~cm}^{-3}\right)$ of $\mathrm{Bi}$ and $\mathrm{P}$ ions before (curve b) and after (curve a) annealing.

\section{$1.1 \times 10^{14} \mathrm{~cm}^{-2} / 30 \mathrm{keV} ; 6.0 \times 10^{13} \mathrm{~cm}^{-2} / 15 \mathrm{keV}$.}

The plateaus with $N_{d}$ lower than $1 \times 10^{20} \mathrm{~cm}^{-3}$ were prepared using the same energies mentioned above but with the doses reduced by a factor of $N_{d} / 1 \times 10^{20}$. The devices were annealed in an inert atmosphere at $600^{\circ} \mathrm{C}$ for 15 min followed by rapid thermal annealing at $950^{\circ} \mathrm{C}$ for $15 \mathrm{~s}$. The samples were analyzed by Rutherford backscattering spectrometry (RBS) using a $1.2 \mathrm{MeV} \mathrm{He}^{+}$beam in random and in $\langle 100\rangle$ aligned (RBS/C) incidence directions.

A resistance bridge was used to measure the resistance of the van der Pauw devices in a ${ }^{4} \mathrm{He}$ cryostat where sample temperature was controlled between $1.7 \mathrm{~K}$ and $300 \mathrm{~K}$. The resistivity was calculated using the expression ${ }^{4}$

$$
\rho=\frac{\pi t}{2 \ln 2}\left(R_{1}+R_{2}\right) f\left(\frac{R_{1}}{R_{2}}\right) .
$$

Here $t$ is the thickness of the sample, and $R_{1}$ and $R_{2}$ are the resistances of two van der Pauw configurations, one rotated by $90^{\circ}$ relative to the other. The van der Pauw function $f\left(R_{1} / R_{2}\right)$ satisfies

$$
\left(\frac{R_{1}-R_{2}}{R_{1}+R_{2}}\right)=\frac{f\left(R_{1} / R_{2}\right)}{\ln 2} \operatorname{arccosh}\left\{\frac{\exp \left[\ln 2 / f\left(R_{1} / R_{2}\right)\right]}{2}\right\} .
$$

For the present samples, $R_{1} / R_{2} \approx 1$, so that the function $f\left(R_{1} / R_{2}\right)$ can be approximated by

$$
f\left(\frac{R_{1}}{R_{2}}\right)=1-\left(\frac{R_{1}-R_{2}}{R_{1}+R_{2}}\right)^{2} \frac{\ln 2}{2} .
$$

\section{RESULTS}

Figure 1 shows the RBS spectra of an annealed sample which has been implanted to the highest doses of $\mathrm{Bi}$ and $\mathrm{P}$ ions $\left(N_{d}=1 \times 10^{20} \mathrm{~cm}^{-3}\right)$. For better visualization, the spectra for backscattering yield of the $\mathrm{Bi}$ atoms were multiplied by a factor of 10. The backscattering of $\mathrm{P}$ atoms is not apparent since these spectra overlap those of $\mathrm{Si}$ atoms. The following features are disclosed from the RBS and RBS/C spectra (respectively, Rutherford Backscattering Spectrometry and RBS with beam aligned to the $\langle 100\rangle$ channel): (i)

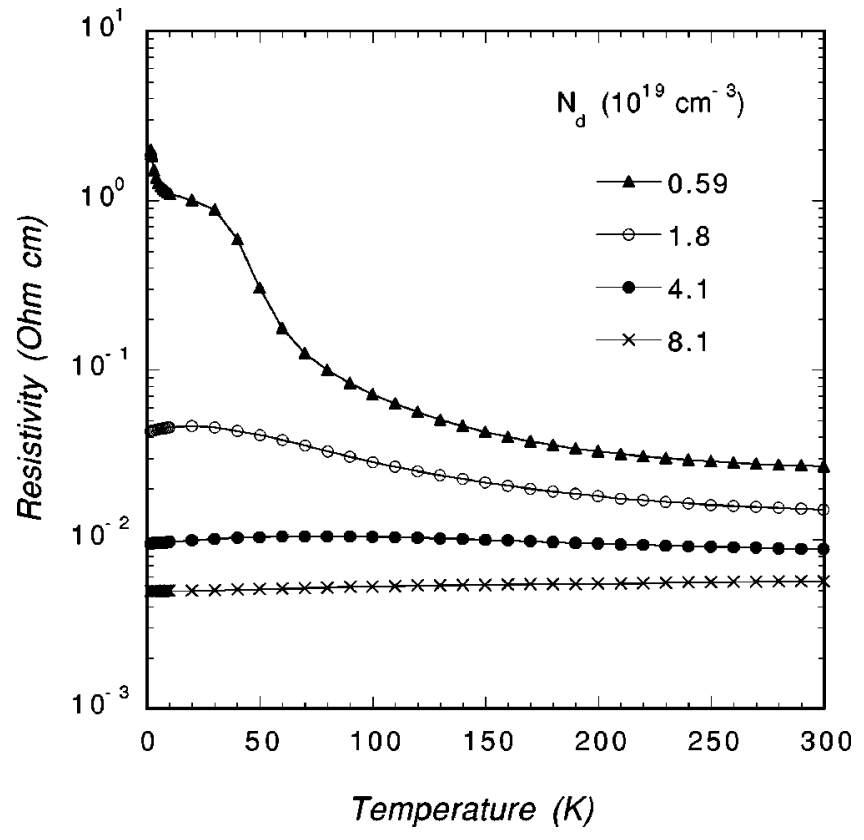

FIG. 2. Temperature dependence of the resistivity of Si:Bi with different donor impurity concentrations $N_{d}$.

the residual defect concentration in the implanted $\mathrm{Si}$ layer is below the sensitivity of the analysis [see Si backscattering yield in curve (a)], (ii) as predicted by TRIM simulation, the Bi concentration depth profile is a plateaulike shape with 5 $\times 10^{19} \mathrm{~cm}^{-3}$ level and depth of $0.25 \mu \mathrm{m}$ [see Bi backscattering yield in curve (b)], (iii) a high substitutional concentration of $\mathrm{Bi}$ is clearly apparent [compare $\mathrm{Bi}$ backscattering yields in curves (b) and (a)]. The spectra of samples with lower $N_{d}$ (not shown) present features similar to those described above.

Figures 2 and 3 show the measured resistivity $\rho$ of the

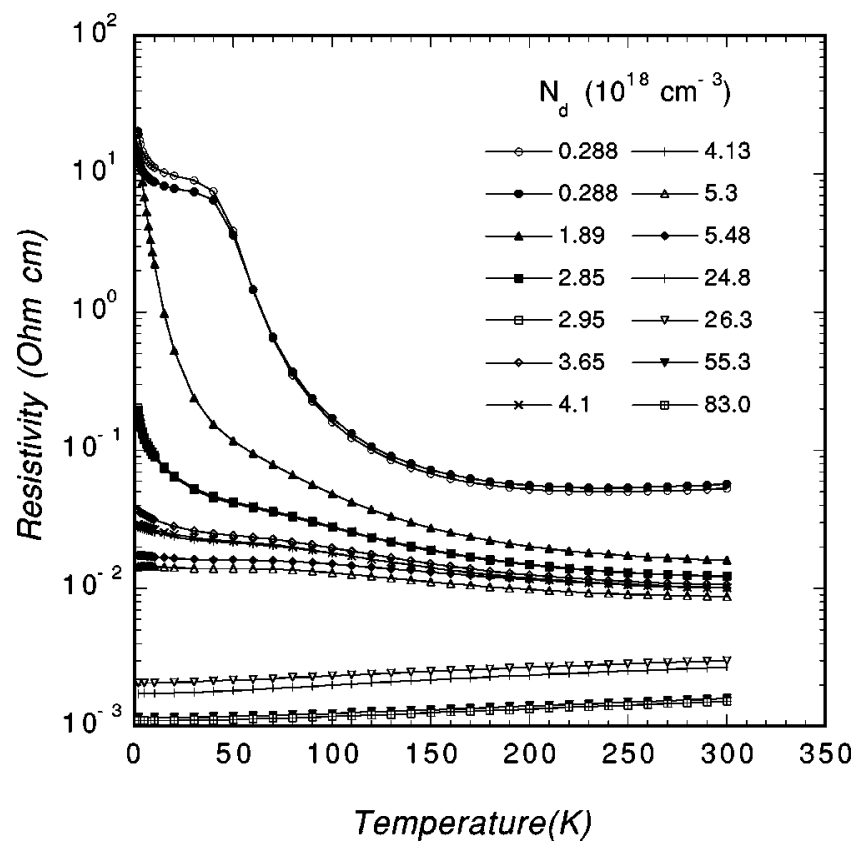

FIG. 3. Temperature dependence of the resistivity of the doubledoped system Si:P,Bi with different donor impurity concentrations $N_{d}$. 


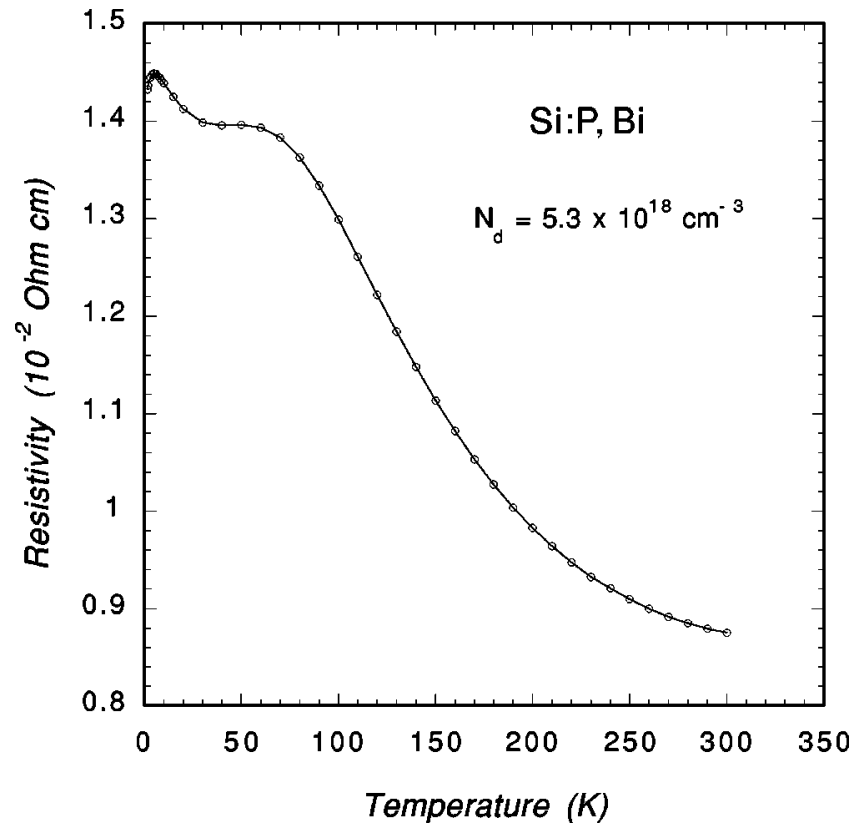

FIG. 4. The resistivity of the Si:P,Bi sample with $N_{d}=5.3$ $\times 10^{18} \mathrm{~cm}^{-3}$ as a function of temperature.

$\mathrm{Si} B \mathrm{Bi}$ and $\mathrm{Si}: \mathrm{P}, \mathrm{Bi}$ systems, respectively, with different donor impurity concentrations $N_{d}$ as a function of temperature. One notices similarities between them. The curves for high impurity concentrations shown at the bottom of the figures clearly display metallic character.

For $\mathrm{Si}: \mathrm{P}, \mathrm{Bi}$, which is the main object of our interest here, Fig. 3 shows the slope of the resistivity curves decreasing with increasing impurity concentration in the region of low temperature down to our lowest temperature, $1.7 \mathrm{~K}$, leading to a smooth decrease in $\rho(T)$ when a MNM transition is expected.

In Figs. 4 and 5 we show the temperature dependence of the resistivity for $N_{d}=5.3 \times 10^{18}$ to $2.63 \times 10^{19} \mathrm{~cm}^{-3}$, corre-

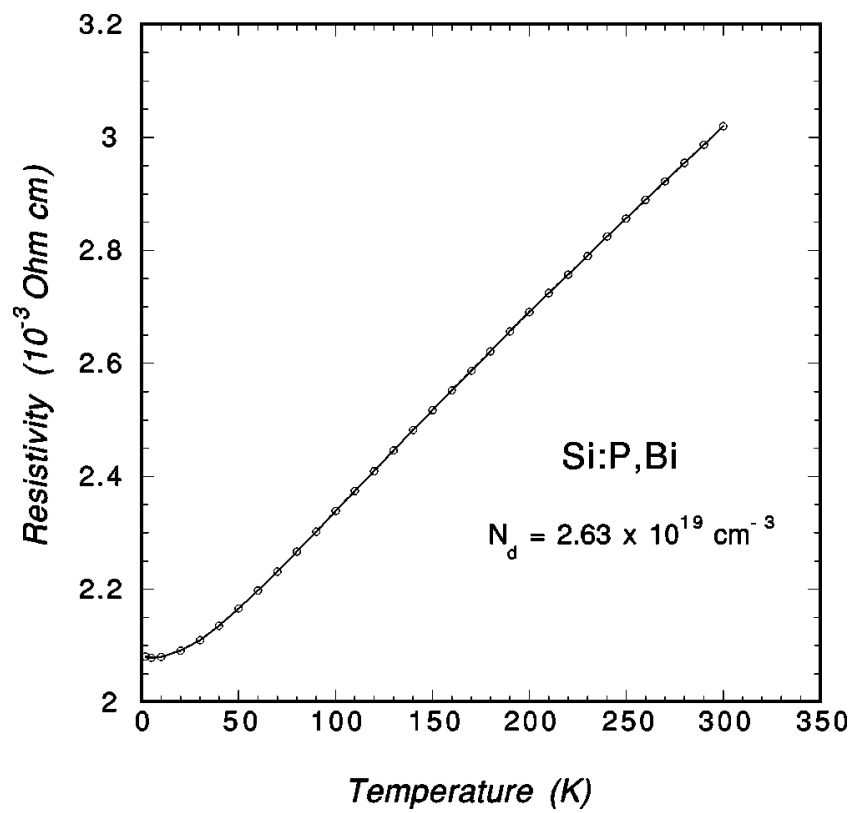

FIG. 5. The resistivity of the Si:P,Bi sample with $N_{d}=2.63$ $\times 10^{19} \mathrm{~cm}^{-3}$ as a function of temperature.

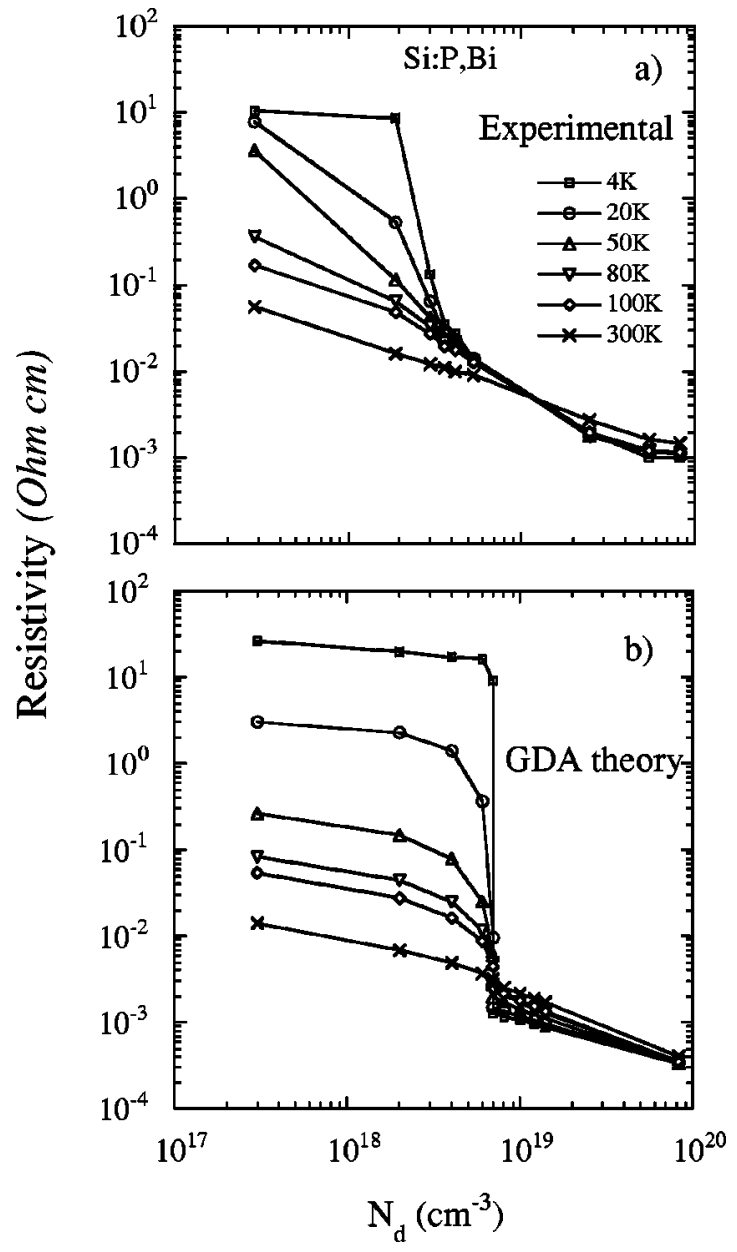

FIG. 6. Resistivity of Si:P,Bi samples as a function of the donor impurity concentration $N_{d}$ (a) measured and (b) calculated by the generalized Drude approach at different temperatures.

sponding to the region of densities where the MNM transition of $\mathrm{Si}: \mathrm{P}, \mathrm{Bi}$ is expected to occur.

Figure 6(a) shows the dependence of the resistivity of $\mathrm{Si}: \mathrm{P}, \mathrm{Bi}$ on the donor impurity concentration $N_{d}$ at different temperatures. One notices that all the curves merge together to one critical point. Figure 6(b) shows the theoretical resistivity calculated from the generalized Drude approach in the same range of donor concentrations and at the same temperatures as Fig. 6(a). The calculation using the GDA method and the determination of the critical concentration of $\mathrm{Si}: \mathrm{P}, \mathrm{Bi}$ are described in the next section.

\section{THEORY AND DISCUSSION}

Following the previous calculations ${ }^{3}$ that use the GDA method to determine the resistivity and the critical concentration of the shallow single-donor system $\mathrm{Si}: \mathrm{Bi}$, we have extended these calculations to double-doped systems, with application to Si:P,Bi. For the resistivity calculations, the results depend on the degree of ionization of the donors. In the metallic region we assume that all donors are ionized. For finite temperatures there are always some ionized donors even on the insulating side of the critical concentration and the density of unionized donors $N_{u}$ differs from the density of donors $N_{d}$. The carriers released should contribute to the 
screening and further reduce the ionization energy, introducing a temperature-dependence, which we will neglect. Assuming that the concentration of each dopant is given by $N_{d}=N_{d, 1}+N_{d, 2}$, we calculate the energy of the two resulting modes, $E_{I, i}$ and the relative weights $\chi_{i}$. The imaginary part of the inverse of the dielectric function in Eq. (8), below, has two peaks corresponding to excitations from the two levels. The relative weights are defined as the relative areas of the two peaks. Some of the dopants will be ionized, $N$, and the rest are unionized. The density of free carriers, in the nonmetallic regime, is determined from simultaneously solving the following equations

$$
\begin{gathered}
N_{d}=N_{u}+N \\
N_{u}=N_{d}\left\{\frac{\chi_{1}}{e^{\left[E_{I, 1}-\mu(N, T)\right] \beta}+1}+\frac{\chi_{2}}{e^{\left[E_{I, 2}-\mu(N, T)\right] \beta}+1}\right\}
\end{gathered}
$$

where $E_{I, i}(i=1,2)$ is the ionization energy for a single donor of type $i, \beta=1 / k_{B} T, k_{B}$ is Boltzmann's constant and $\mu(N, T)$ is the chemical potential. The model is then presented with two localized donor levels below the conduction band. The carriers are distributed among these levels and the continuum states according to the Fermi-Dirac distribution function.

For nonpolar semiconductors the generalized Drude approach for the resistivity is reduced to ${ }^{2,3,9-11}$

$$
\rho(\omega)=\frac{-i m^{*} \omega}{N e^{2}}+\frac{i 2}{3 \pi N \omega} \int_{0}^{\infty} q^{2}\left[\frac{1}{\varepsilon_{T}(q, \omega)}-\frac{1}{\varepsilon_{T}(q, 0)}\right] d q,
$$

where $e$ is the electric charge, $m^{*}$ is the effective mass, and $\varepsilon_{T}$ is the total dielectric function. We have assumed a random distribution of Coulomb impurities. The total dielectric function is given by ${ }^{10}$

$$
\varepsilon_{T}(q, \omega)=\varepsilon+v \alpha_{1}(q, \omega)+i v \alpha_{2}(q, \omega),
$$

where $\varepsilon$ is the dielectric constant for $\mathrm{Si}$ and $\alpha_{1}$ and $\alpha_{2}$ are the real and imaginary parts of the polarizabilities from one of the $v$ valleys of dopant carriers. The number of valleys is $6, \varepsilon=11.4 \mathrm{in} \mathrm{Si}$, and these functions are temperature dependent. The imaginary part can be obtained analytically in the random phase approximation. ${ }^{12}$ It is given by

$\alpha_{2}(Q, W)$

$$
\begin{aligned}
= & -\frac{m^{*} e^{2}}{8 \hbar k_{F} Q^{3} B} \\
& \times\left[\ln \left(\frac{\cosh \left\{B\left[W+\left(Q^{2}+W^{2} / Q^{2}-M\right) / 2\right]\right\}}{\cosh \left\{B\left[W-\left(Q^{2}+W^{2} / Q^{2}-M\right) / 2\right]\right\}}\right)-2 B W\right],
\end{aligned}
$$

where we have introduced the dimensionless variables $Q$ $=q / 2 k_{F}, \quad W=\hbar \omega / 4 E_{F}, \quad B=\beta E_{F}$, and $M=\mu / E_{F}$. The quantity $k_{F}$ is the Fermi wave vector, given by $k_{F}$ $=\left(3 N \pi^{2} / \nu\right)^{1 / 3}$, and $E_{F}$ is the Fermi energy. The real part can be obtained from the imaginary part through the Kramers-Kronig dispersion relation.
We are interested in the static resistivity, which can be written as

$$
\rho(0)=\frac{16 \hbar k_{F}^{3} v}{12 \pi N E_{F}} \int_{0}^{\infty} Q^{2} \frac{\left.\left[\partial \alpha_{2}(Q, W) / \partial W\right]\right|_{W=0}}{\left[\varepsilon+v \alpha_{1}(Q, 0)\right]^{2}} d Q,
$$

which can be reduced to

$$
\rho(0)=\frac{2 v\left(m^{*} e\right)^{2}}{3 \pi N \hbar^{3} k_{F}} \int_{0}^{\infty} \frac{\left\{1-\tanh \left[0.5 B\left(Q^{2}-M\right)\right]\right\}}{Q\left[\varepsilon+v \alpha_{1}(Q, 0)\right]^{2}} d Q .
$$

The chemical potential $\mu$ is obtained from the implicit expression $^{3,11,13}$

$$
B^{3 / 2}=\int_{0}^{U} \frac{3 y}{1-y^{2}}\left\{A+\ln \left[\left(1-y^{2}\right) / y^{2}\right]\right\}^{1 / 2} d y,
$$

where $U=\left(1+e^{-A}\right)^{-1 / 2}$ and $A=B M=\mu B$. For a given $A$, one obtains $\mathrm{B}$ leading to a relation between them.

The calculated resistivity of $\mathrm{Si}: \mathrm{P}, \mathrm{Bi}$ as a function of impurity concentration and temperature obtained using the procedure described above is presented in Fig. 6(b) and compared to the measured resistivity shown in Fig. 6(a). Both measured and calculated resistivity curves present similar forms, converging to a value around $6.0 \times 10^{18} \mathrm{~cm}^{-3}$, which is determined to be the critical concentration $N_{c}$ for the MNM transition in Si:P,Bi. For the measured resistivity at $300 \mathrm{~K}$ we observe a small difference compared to the other ones. For impurity concentrations in the metallic region, i.e., above $N_{c}$, the temperature dependence of the resistivity is negligible in comparison with the nonmetallic region.

The ionization energies $E_{I, i}$ for the double-doped systems are determined using the Lorentz expression for the dielectric function but with the Lorentz-Lorenz correction and two ionization energies. The dielectric function for silicon plus unionized donors is

TABLE I. Theoretical $(t)$ and experimental $(e)$ results for the critical concentration $N_{c}$ for the MNM transition.

\begin{tabular}{lccc}
\hline \hline System & $E_{I}(\mathrm{mev})$ & $N_{c}^{t}\left(10^{18} \mathrm{~cm}^{-3}\right)$ & $N_{c}^{e}\left(10^{18} \mathrm{~cm}^{-3}\right)$ \\
\hline Si:Bi & 71.00 & 16.90 & $\sim 20.00^{\mathrm{a}}$ \\
Si:As & 53.60 & 7.26 & $8.50^{\mathrm{b}}$ \\
Si:P & 45.44 & 4.42 & $3.48^{\mathrm{d}}$ \\
& & & $3.75^{\mathrm{c}}$ \\
Si:P,Bi & & 7.00 & $\sim 6.00^{\mathrm{e}}$ \\
& & $\sim 6.00^{\mathrm{f}}$ & \\
Si:P,As & & 5.49 & $5.10^{\mathrm{g}}$ \\
\hline \hline
\end{tabular}

${ }^{\mathrm{a}}$ From Ref. 3.

${ }^{b}$ From Ref. 14.

${ }^{\mathrm{c}}$ From Ref. 15.

${ }^{\mathrm{d}}$ From Ref. 16.

${ }^{\mathrm{e}}$ From Fig. 6(a).

${ }^{\mathrm{f}}$ From Fig. 6(b).

${ }^{\mathrm{g}}$ From Ref. 1. 


$$
\varepsilon(\omega)=1+\frac{\frac{4 \pi N_{u, 1} \alpha_{d, 1} \omega_{0,1}^{2}}{\omega_{0,1}^{2}-\omega^{2}-i \Gamma_{1} \omega}+\frac{4 \pi N_{u, 2} \alpha_{d, 2} \omega_{0,2}^{2}}{\omega_{0,2}^{2}-\omega^{2}-i \Gamma_{2} \omega}+4 \pi N_{h} \alpha_{h}}{1-\frac{1}{3}\left(\frac{4 \pi N_{u, 1} \alpha_{d, 1} \omega_{0,1}^{2}}{\omega_{0,1}^{2}-\omega^{2}-i \Gamma_{1} \omega}+\frac{4 \pi N_{u, 2} \alpha_{d, 2} \omega_{0,2}^{2}}{\omega_{0,2}^{2}-\omega^{2}-i \Gamma_{2} \omega}+4 \pi N_{h} \alpha_{h}\right)},
$$

where $\alpha_{d, i}$ is the static polarizability for a single donor of type $i$ in the silicon matrix, $\alpha_{h}$ is the contribution from the static polarizability for the host, $N_{u, i}$ the density of unionized donors, $N_{h}$ is the contribution of the host charge density and $E_{0, i}=\hbar \omega_{0, i}$ is the ionization energy for a single donor of type $i$. Following a procedure similar to that used in Ref. 3, the denominator of the dielectric function has two zeros, corresponding now to the two ionization energies. As the donor concentration increases, one of the ionization energies eventually vanishes. This defines the critical concentration. The calculated and experimental values of MNM transitions are presented in Table I.

\section{SUMMARY}

We have reported results of an experimental and theoretical investigation of the impurity resistivity of the ionimplanted double-donor system $\mathrm{Si}: \mathrm{P}, \mathrm{Bi}$ from room tempera- ture down to $1.7 \mathrm{~K}$ for concentrations spanning the insulating to the metallic regimes. Good agreement with the experimental results were obtained from calculations using a generalized Drude approach. The value of the impurity critical concentration $N_{c}$ for the MNM transition was estimated from both methods above as well as through the dielectric function with the Lorentz-Lorenz correction. The critical impurity concentration $N_{c}$ for the double-doped Si:P,Bi system was found to be intermediate between the critical concentrations of the two single-doped systems, Si:P and $\mathrm{Si}: \mathrm{Bi}$.

\section{ACKNOWLEDGMENTS}

A.F.S., J.P.S., and H.B. would like to thank the Brazilian National Research Council (CNPq), and B.E.S. the Swedish Natural Science Research Council (NFR) for financial support. The work of H.Z. and M.P.S. was supported by the U.S. Department of Energy Grant No. DE-FG02-84ER45153.
${ }^{1}$ P. F. Newman and D. F. Holcomb, Phys. Rev. Lett. 51, 2144 (1983).

${ }^{2}$ A. Ferreira da Silva, Bo E. Sernelius, J. P. de Souza, and H. Boudinov, J. Appl. Phys. 79, 3453 (1996).

${ }^{3}$ E. Abramof, A. Ferreira da Silva, Bo E. Sernelius, J. P. de Souza, and H. Boudinov, Phys. Rev. B 55, 9584 (1997).

${ }^{4}$ L. J. Van der Pauw, Philips Res. Rep. 13, 1 (1958).

${ }^{5}$ F. A. Trumbore, Bell Syst. Tech. J. 39, 205 (1960).

${ }^{6}$ J. F. Ziegler, J. P. Biersak, and U. Littmark, The Stopping and Ranges of Ions in Solids (Pergamon, New York, 1985), Vol I.

${ }^{7}$ J. P. de Souza and P. F. Fichtner, J. Appl. Phys. 74, 119 (1993).

${ }^{8}$ J. P. de Souza and D. K. Sadana, in Handbook on Semiconductors, edited by S. Mahajan (North-Holland, Amsterdam, 1994), Vol. 36, p. 2036.

${ }^{9}$ Bo E. Sernelius, Phys. Rev. B 41, 3060 (1990).
${ }^{10}$ Bo E. Sernelius, Phys. Rev. B 36, 1080 (1987).

${ }^{11}$ Bo E. Sernelius and E. Soderstrom, J. Phys.: Condens. Matter 3, 1493 (1991).

${ }^{12}$ R. Sirko and D. L. Mills, Phys. Rev. B 18, 4373 (1978).

${ }^{13}$ Bo E. Sernelius, in Shallow Impurities in Semiconductors, Proceedings of the Third International Conference on Shallow Impurities in Semiconductors, edited by B. Monemar, IOP Conference Proceeding No. 95 (Institute of Physics and Physical Society, London, 1988), p. 137.

${ }^{14}$ P. F. Newman and D. F. Holcomb, Phys. Rev. B 28, 638 (1983).

${ }^{15}$ P. Dai, Y. Zhang, and M. P. Sarachik, Phys. Rev. B 49, 14039 (1994).

${ }^{16}$ T. F. Rosenbaum, K. Andres, G. A. Thomas, and R. N. Bhatt, Phys. Rev. Lett. 45, 1723 (1980). 